Indian J. Anim. HIth. (2019), 58(1) : 143-146

DOI: https://doi.org/10.36062/ijah.58.1.2019.143-146

\title{
CRISPR-Cas GENOME EDITED TWIN CAN FIGHT AGAINST AIDS AND POSSIBLY WIN
}

\author{
T. K. GoswAMI \\ Immunology Section \\ Indian Veterinary Research Institute \\ Izatnagar-243 122, Bareilly,UP, India
}

Can we forecast our future eventful fate with the available information from gene sequencing data as the astrologers usually predict. Scientifically both are similar but not same. Human endeavor is not only to predict rather can modify the gene known as gene editing. Any untoward event which may arise due to the inherited gene in human genome can be tamed down by gene editing technique. Recently biotechnology has been applied to modify the genome of human embryo as per their wish. Simply we can literally say it is a designer kid produced out of gene editing in parental gene therefore offspring is modified. To alter the gene in human zygote is possible yet there is stringent rule and ethics prevailed. Our discussion is focused about the recent announcement in gene editing technique by Chinese Scientist He Jiankui; on $28^{\text {th }}$ November 2018. It has revolutionized the human society so that in near future we may find parents can design their child with specific character according to their need. Parents can ask to customize their own child before implanting the zygote in mother's womb so that new born will be resistant to a particular disease or will avoid certain physical illness in its future life for which it has been designed. Contrary to it, normal child may suffer even succumb with that particular disease. This has been possible due to CRISPR-Cas gene editing technique. This technique acts like scissors for cutting the DNA at a specific spot and next stitch a new piece of DNA at that spot or delete a stretch of DNA and again rejoin both the ends with accuracy. One can say it is similar like a job of a tailor to cut and stitch a cloth for dress designing. It is imperative to elaborate the technique for the readers. Idea is that once we remove a stretch of DNA the rest of the DNA with the house keeping genes will operate without interruption to maintain the house. Similarly when a stretch of DNA is deleted and replaced by a new stretch of DNA, the resulting DNA should express the desired effect coded for by that new DNA without affecting the characteristic of that cell. The CRISPR-Cas system is the inherited system in bacteria to cleave the foreign DNA. 
Bacteria usually acquire foreign DNA due to conjugation, transformation and transduction, these DNA can cause threat to bacteria. Phage is a type of virus which is obligatory parasite for bacteria. Once get entry inside the host, phage DNA are subjected to the action of host nuclease and fragmented. This is one of the self-defense immune mechanisms of bacteria. Beyond the rule occasionally bacteria allow exogenous DNA to be incorporated in its own DNA and conserve the foreign DNA that has advantages to the host. It has been found that bacteria harbor a specific gene clustered known as CRISPR (Clustered Regularly Interspaced Short Palindromic Repeats) that ultimately provides acquired immunity against virus and plasmid. Short sequences of non-self genetic material derived from invading phage DNA or from plasmid DNA are first recognized by the bacteria and it is incorporated into the host genome between short DNA repeats known as spacer. The spacers are used as recognition elements to recognize the new virus genome to be destroyed. By adding new spacer, new virus can be recognized. New spacers are added at one side of the CRISPR, so that it is added one after another representing the chronological record of the viruses the cell have encountered. The CRISPR-Cas system targets DNA or RNA and other mobile genetic elements. Since the genome is modified due to acquisition of spacer, subsequently it is transferred to next generation so that offspring is protected
(Rath et al., 2015). It is distinguished by clustered regulatory interspaced short palindromic repeats. The captured DNA segments (spacers) from invading viruses are used to create DNA segments known as CRISPR arrays. The DNA spacer is transcribed into non coding RNAs (noncoding RNAs don't translate into protein). Once there is a close match between the sequence of foreign DNA and transcribed noncoding RNAs; next steps proceed so that non-coding RNA along with specific Cas protein can bind to incoming foreign invaders DNA. The Cas proteins are coded for by Cas gene located vicinity to CRISPR loci. Cas protein having enzymatic action to cleave the DNA (Bhaya et al., 2011). The CRISPR arrays enable bacteria to remember the viruses or closely related viruses so that once the same virus attack again the bacteria produce RNA segment from CRISPR arrays to target the virus DNA to silence them (Horvath and Barrangou, 2010). The bacteria use Cas 9 or similar enzymes to chew the DNA and thereby invading virus become noninfective. The RNA also binds to the Cas-9 enzyme. Therefore the RNA once recognize the specific DNA sequence both DNARNA binding takes place; simultaneously the Cas- 9 enzyme already in association with RNA cuts the DNA at target location. This immune mechanism of bacteria has been adapted in laboratory using CRISPRCas system by the scientist to cleave the DNA for insertion or deletion of desired gene in human genome. 
During second International Summit on human genome editing held in Hong Kong on November 28, 2018, He Jiankui (spell as HEEH JEE-an-qway) and his group have announced the first time use of CRISPRCas9 genome editing in human embryo that resulted in birth of twin sister Lulu \& Nana resistant to HIV infection ( $\mathrm{Li}$ et al., 2019). These groups edited the gene responsible for expression of chemokine receptor designated as CCR5 (CysteineCysteine chemokine receptor). Chemokines are low molecular weight proteins that bind to the chemokine receptors expressed on hematopoietic cell to exert its physiological effect. CCR5 is one of the members of chemokine receptor family. Chemokine receptors have specific physiological role and never designed to receive any virus. However the virus hijacks this receptor for its own benefit thereby the CCR5 forms a protein doorway to enter the CD4 T-cells. Chinese scientist He Jiankui announced that using CRISPR-Cas9 gene editing technique he deleted both the copies of CCR5 gene in human embryos. Both the copies indicate that each cell harbor one set of gene from each parent (father and mother) therefore deletion of one set of gene will not be fruit full as the remaining set will compensate the loss. In the work proper eight HIV positive male individual volunteers were taken in the study whereas ovum donors were HIV negative. The sperm was thoroughly washed and injected in to the collected eggs from female volunteers. In the fertile zygote the gene responsible for CCR5 was edited by CRISPR-Cas technique and the outcome was successful delivery of twin girl. The twin was named as Lulu and Nana. Normal citizen of any country are not interested to know the intricacy of the CRISPR-Cas technique rather more concerned about the fate of the new born performance in its future life. In his trial He Jiankui edited 30 embryos and implanted two embryos in the mothers" womb that resulted delivery of twin girl without any abnormality. For ethical issue the privacy of this twin has been maintained. It is not wise to disable CCR5 gene as it has key role to maintain the cell physiology suggested by the critics. Further HIV is highly mutable virus and CCR5 is only one of the co-receptor for which HIV has preferential choice to bind. Scientific community is divided in their opinion to support this technique to edit human embryo (Daley et al., 2019). It has received enough criticism throughout the world yet one has to admit that CRISPR-Cas technique has great potential for gene editing which will be a boon to human society. Scientific community from all over the world has expressed their concern over human gene editing which may invite a serious threat to human society by which a ban has been imposed so that presently $\mathrm{He}$ Jiankui is not permitted to work in this aspect. In comparison to available gene editing techniques like zinc-finger nucleases (XFNs) and transcription activators nucleases (TALENs), the CRISPR-Cas technique is faster and less expensive and more precise. Negative point is that targeting efficiency is still inadequate 
in CRISPR-Cas technique (Baumann, 2016). CRISPR-Cas technology adapted for gene editing in human embryo is now ethically charged, because changes in human embryo can be transmitted to next generation eventually affect the subsequent population is a serious issue that may spoil the entire gene pool in human society (National Academies of Sciences, Engineering and Medicine, 2017). Application of CRISPR-Cas technique to rectify single-gene disorder like cystic fibrosis, hemophilia and sickle cell disease has been explored to prove its efficacy in research. Previously gene editing has been confined to somatic cells

\section{REFERENCES}

Baumann M, 2016. CRISPR/Cas9 genome editing — new andold ethical issues arising from a revolutionary technology. Nanoethics, 10(2): 139-159

Bhaya D, Davison M and Barrangou R, 2011. CRISPR-Cas system in bacteria and Archaea: versatile small RNAs for adaptive defense and regulation. Annu Rev Genet, 45: 273-297

Daley GQ, Lovell-Badge R and Steffann J, 2019. After the storm responsible path for genome editing. N England J M, doi: 10.1056/NEJMp1900504

Horvath P and Barrangou R, 2010. CRISP/Cas, the immunesystem of bacteria and archaea. Science, 327(5962): 167-170

Li Z, Walker S, Nie J and Zhang X, 2019. within the lab but scientific ethics does not permit for gene editing in sperm, ovum or in embryo as the character may pass to next generation that is never welcomed. Whether ethics is imposed or not but academic community should apply gene editing in human embryo with respect and dignity towards human life. As reported recently scientist community under Chinese academy of engineering are concerned about the wellbeing of the infant twins born out of gene editing technique (Zhang et al., 2019). They have urged the scientific community to provide preventive measurement for potential health and social issues related to the genome editing

Experiments that led to the first geneedited babies: the ethical failings and the urgent need for better governance. J Zhejiang 32 Univ-Sci B (Biomed \& Biotechnol), 20(1): 32-38

National Academies of Sciences, Engineering and Medicine, 2017. Human Genome Editing: Science Ethics and Governance. Washington DC: The National Academies Press

Rath D, Amlinger L, Rath A and Lundgern M, 2015. The CRISPR-Cas immune system: Biology, mechanisms and applications. Biochimie, 117: 119-128

Zhang B, Chen Z, Yi J, Tang H and Wang C, 2019. Chinese academy of engineering calls for actions on the birth of gene-edited infants. Lancet, 393(10166): 25 